\title{
75 Jahre Raumforschung und Raumordnung
}

\author{
Hans Heinrich Blotevogel
}

Online publiziert: 14. Januar 2011

(C) Springer-Verlag 2010

Im Jahre 1936, also vor 75 Jahren, erschien ,Raumforschung und Raumordnung“ (RuR) zum ersten Mal. Im ersten Heft wurde die neue Zeitschrift angekündigt als ,Monatsschrift der Reichsarbeitsgemeinschaft für Raumforschung“(RAG). Als Herausgeber fungierte Konrad Meyer, Professor für Ackerbau und Landbaupolitik an der Universität Berlin und zugleich Obmann der RAG, die als Netzwerk von raumwissenschaftlich arbeitenden Hochschullehrern der 1935 gegründeten staatlichen „Reichsstelle für Raumordnung“ zuarbeiten sollte.

Dass die Zeitschrift in diesem Jahr nicht im 75., sondern im 69. Jahrgang erscheint, hängt mit der Unterbrechung infolge des Zweiten Weltkriegs und der unregelmäßigen Erscheinungsweise in den ersten Nachkriegsjahren zusammen. Dass dennoch eine gewisse Kontinuität bestand, geht zurück auf Kurt Brüning, der als letzter RAG-Obmann im Winter 1944/45 die RAG von Berlin nach Hannover und Göttingen verlagerte und sie in die Akademie für Raumforschung und Landesplanung (ARL) umwandelte. Als erster Präsident der ARL sorgte er dafür, dass RuR ab 1948 unter demselben Namen fortgeführt wurde.

Von 1953 bis 2009 wurde RuR gemeinsam von der ARL und dem von der Bundesregierung getragenen Institut für Raumforschung (IfR) bzw. seinen Nachfolgeorganisationen Bundesforschungsanstalt für Landeskunde und Raumordnung (BfLR), Bundesamt für Bauwesen und Raumordnung (BBR) bzw. Bundesinstitut für Bau-, Stadtund Raumforschung (BBSR) herausgegeben. In diesen Jahrzehnten entwickelte sich RuR zu der führenden deut-

Prof. Dr. H. H. Blotevogel $(\bowtie)$

Leibniz-Forum für Raumwissenschaften,

Akademie für Raumforschung und Landesplanung,

Hohenzollernstraße 11, 30161 Hannover, Deutschland

E-Mail: hans.blotevogel@tu-dortmund.de schen Fachzeitschrift für Fragen der Raumentwicklung und Raumplanung.

2010 erfuhr RuR eine neue Profilierung als forschungsorientierte raumwissenschaftliche Zeitschrift, verbunden mit einem Wechsel der Herausgeber und des Verlags. Das BBSR zog sich zurück, und seitdem wird RuR gemeinsam von den fünf raumwissenschaftlichen Forschungseinrichtungen herausgegeben, die der Leibniz-Gemeinschaft angehören bzw. ihr assoziiert sind.

Soweit in wenigen Stichworten die äußerliche Seite der Entwicklung von RuR. Sie ist gut dokumentiert, während eine systematische und kritische Aufarbeitung der inhaltlichen Entwicklung noch aussteht. Die RuR-Herausgeber beabsichtigen, hierzu eine Reihe von Forschungsarbeiten anzuregen, um die Entwicklungslinien der Zeitschrift nicht nur zu dokumentieren, sondern auch kritisch zu reflektieren und sie sowohl in ihren planungs- als auch zeitgeschichtlichen Kontexten zu analysieren.

Aber lohnt es sich überhaupt, vertiefte Blicke auf die inhaltliche Entwicklung von RuR zu werfen? Genügt es nicht, wenn die nunmehr 68 abgeschlossenen Jahrgänge für Interessenten in Fachbibliotheken zugänglich sind? Und überhaupt: Kann die Beschäftigung mit der Vergangenheit überhaupt einen nennenswerten Nutzen für uns heute stiften?

Zunächst einmal: 75 Jahre RuR bieten einen einmaligen Quellenfundus zur Geschichtsschreibung der Raumplanung seit der Etablierung der staatlichen Raumordnung im nationalsozialistischen Staat (1935). In der Zeitschrift spiegeln sich personelle und ideelle Kontinuitäten und Brüche wie in kaum einer anderen planungsgeschichtlichen Quelle wider. Dabei hat die neuere planungsgeschichtliche Forschung gezeigt, dass die Kontinuitäten über die vermeintliche Zäsur 
von 1945 hinweg entschieden größer waren als bisher vielfach angenommen. ${ }^{1}$

„Raumforschung und Raumordnung“ war stets ein politisches Projekt, wobei die Politiknähe im Laufe der Geschichte der Zeitschrift allerdings erheblich variierte. In seinem Geleitwort zum ersten RuR-Heft 1936 erklärte der nationalsozialistische Wissenschafts- und Kultusminister Bernhard Rust unmissverständlich: „Der Ansatz der Wissenschaft für die Raumforschung und Raumordnung wird sie zwangsläufig hineinführen in die Kernfragen des Nationalsozialismus: Blut und Boden, Volk und Raum", und der spiritus rector von RAG und RuR Konrad Meyer umriss im selben Heft in einem programmatischen Artikel „Raumforschung" als eine neue normative Wissenschaft im Geiste des Nationalsozialismus. ${ }^{2}$

Ist dieses dirty little secret der Raumwissenschaften und Raumplanung für uns heute belanglos? Oder sind die zentralen Begriffe wie „Raum“ und Theoreme wie „Tragfähigkeit“, „Dezentralisierung“, „Zentraler Ort“" usw. gleichsam hoffnungslos ideologisch kontaminiert? Wie ist die Gemeinschaft der Raumforscher und Raumordner nach

\footnotetext{
${ }^{1}$ Leendertz, A. (2008): Ordnung schaffen. Deutsche Raumplanung im 20. Jahrhundert. Göttingen. - Mäding, H.; Strubelt, W. (Hrsg.) (2009): Vom Dritten Reich zur Bundesrepublik. Beiträge einer Tagung zur Geschichte von Raumforschung und Raumordnung. Hannover. = Arbeitsmaterial der ARL, Nr. 346.

${ }^{2}$ Meyer, K. (1936): Raumforschung. In: Raumforschung und Raumordnung 1, 1, 2-4.
}

1945 mit ihrem politisch kompromittierten Erbe umgegangen? Erfolgte eine aktive Auseinandersetzung, oder wurde die personelle und ideologische Verstrickung beschwiegen, verdrängt, vertuscht und beschönigt? Lässt sich jenseits der nationalsozialistischen Raumforschung und Raumordnung so etwas wie eine ,gute Tradition“ der raumwissenschaftlichen Forschung - sei es im politisch neutralen Sinne oder als explizit normative Handlungswissenschaft im demokratischen Staat - identifizieren?

Diese und weitere Fragen führen über die rein wissenschafts- und planungsgeschichtliche Perspektive weit hinaus. Sie verweisen auf zentrale Fragen des Selbstverständnisses der anwendungsorientierten Raumwissenschaften und der Planungsforschung, also derjenigen Fachrichtungen, denen sich die raumwissenschaftlichen Einrichtungen der LeibnizGemeinschaft verpflichtet fühlen und welche das Selbstverständnis von RuR heute prägen. Die kritische Befassung mit der eigenen Geschichte dient insofern stets auch der aktuellen Selbstvergewisserung. Dass dies nicht im Sinne einer apologetischen Traditionspflege, sondern als kritische Auseinandersetzung geschieht, dürfte sich bei der spezifischen Geschichte von RuR von selbst verstehen.

Seriöse Forschungsarbeiten benötigen Zeit. Sobald qualifizierte Manuskripte vorliegen, wird RuR sie publizieren und damit nicht nur die lange verschleppte Auseinandersetzung mit der eigenen Geschichte ein Stück vorantreiben, sondern hoffentlich auch Beiträge zu dem nicht minder dringlichen Diskurs über das Selbstverständnis von Raumwissenschaften und Raumplanung heute leisten. 